\title{
Remaining useful tool life predictions in turning using Bayesian inference
}

\author{
Jaydeep M. Karandikar ${ }^{1}$, Ali Abbas², and Tony L. Schmitz ${ }^{3}$ \\ ${ }^{1,3}$ Mechanical Engineering and Engineering Science, University of North Carolina at Charlotte, Charlotte, NC, 28262, USA \\ jkarand1@uncc.edu \\ tony.schmitz@unccc.edu \\ ${ }^{2}$ Industrial and Systems Enterprise Engineering, University of Illinois at Urbana-Champaign, Urbana, IL, 61801, USA \\ aliabbas@illinois.edu
}

\begin{abstract}
Tool wear is an important factor in determining machining productivity. In this paper, tool wear is characterized by remaining useful tool life in a turning operation and is predicted using spindle power and a random sample path method of Bayesian inference. Turning tests are performed at different speeds and feed rates using a carbide tool and MS309 steel work material. The spindle power and the tool flank wear are monitored during cutting; the root mean square of the time domain power is found to be sensitive to tool wear. Sample root mean square power growth curves are generated and the probability of each curve being the true growth curve is updated using Bayes' rule. The updated probabilities are used to determine the remaining useful tool life. Results show good agreement between the predicted tool life and the empirically-determined true remaining life. The proposed method takes into account the uncertainty in tool life and the growth of the root mean square power at the end of tool life and is, therefore, robust and reliable.
\end{abstract}

\section{INTRODUCTION}

Tool wear can impose a significant limitation to machining productivity, especially in hard-to-machine materials such as titanium and super nickel alloys. Although models exist for tool life estimation, such as the Taylor tool life equation (Taylor, 1906), extended Taylor-type tool life equation (Tlusty, 2000), temperature-based tool life models (Quinto, 1988), and others (Jawahir, Gosh, \& Exner, 1995 and Stein, Gosh, \& Jawahir, 1997), tool wear is widely considered to be stochastic and difficult to predict. This is primarily due to tool-to-tool performance variations, process variations, and factors not included in the models. Incorrect tool life

Karandikar et al. This is an open-access article distributed under the terms of the Creative Commons Attribution 3.0 United States License, which permits unrestricted use, distribution, and reproduction in any medium, provided the original author and source are credited. estimations directly affect process efficiency. In this work, the limitation to machining productivity imposed by tool wear is addressed using Bayesian inference techniques. A new approach that establishes an estimate of the remaining useful life $(R U L)$ for a selected tool based on the time domain root mean square (RMS) spindle power during machining is described.

A tool condition monitoring system is used to determine when a tool change is necessary. Implementation is composed of three steps: 1) identify and extract relevant features correlated to tool wear; 2) train the system using tool wear experiments; and 3) develop an intelligent inference technique for predicting tool wear (Dimla, 2000 and Prickett, \& Johns, 1999). Tool condition monitoring systems which rely on mathematical models generally requires a significant amount of empirical data and, therefore, are challenging to apply in industrial applications (Dimla, 2000). Another important limitation to tool condition monitoring is the stochastic nature of the sensor signal due to non-homogeneities in the workpiece, for example (Constantinides, \& Bennett, 1987). Therefore, a tool condition monitoring system which relies on a deterministic threshold value may not be reliable due to the uncertainty in the value at the end of tool life. The proposed method takes into account both the inherent uncertainty in tool life and the threshold value (for the selected sensor) at the end of tool life. Research has shown acoustic emission, vibration signals, cutting forces (static and dynamic), and spindle power/current to be sensitive to tool wear; a review of the sensor signals is presented in (Dimla, 2000). In this work, spindle power is used for tool condition monitoring since it is non-intrusive (Ravindra, Srinivasa, \& Krishnamurthy, 1993 and Rangawal, \& Dornfield, 1990) and offers a lower cost alternative to the measurement of most other relevant physical quantities.

Although Bayesian methods have gained popularity in recent years, their application to tool condition monitoring 
has been limited in the literature. Elangovan, Ramachandran, and Sugmaran (2010) used a combination of Naïve Bayes and Bayes' network for tool condition monitoring in a discrete case. Dey and Stori (2005) used Bayesian networks for root cause analysis of process variations. Karandikar, Schmitz, and Abbas (2010) used Bayesian inference for tool life predictions in pre-process planning stage. The primary contribution of this paper is to demonstrate and validate a novel random sample path method of Bayesian inference for remaining useful tool life predictions.

The remainder of the paper is organized as follows. First, an overview of Bayesian inference is provided. Second, training experiments are described that identify the influence of tool wear on spindle power and the corresponding tool life. The training experiment data was used to determine the prior, or initial beliefs. Third, the random sample path method for Bayesian updating is described. Fourth, additional experiments are detailed and the remaining useful tool life is predicted using the measured root mean square power data. Finally, remaining useful tool life predictions are compared to the true remaining life.

\section{BAYESIAN INFERENCE}

As noted, tool life is considered stochastic and, in general, difficult to predict. This is due to many factors such as the complex nature of the tool wear phenomenon and tool-totool performance variation. Therefore, tool life should be characterized by a probability distribution to incorporate its inherent uncertainty. A Bayesian model treats an uncertain quantity as a random (or uncertain) variable using a probability distribution. It provides a normative and rational method for updating beliefs when new information is available. Let the prior distribution about an uncertain event, $A$, be $\mathrm{P}(A)$; the likelihood of obtaining an experimental result $B$ given that event $A$ occurred be $\mathrm{P}(B \mid A)$, and the probability of receiving experimental result $B$ (without knowing that $A$ has occurred) be $\mathrm{P}(B)$. Bayes' rule is used to determine the posterior probability about event $A$ after observing the experiment results, $\mathrm{P}(A \mid B)$, as shown in Eq. 1.

$$
P(A \mid B)=\frac{P(B \mid A) P(A)}{P(B)}
$$

The product of the prior and likelihood function is used to calculate the posterior distribution. For multiple measurements, the posterior distribution after the first measurement, or update, becomes the prior for the second, and so on. An important requirement for applying Bayes' rule in this case is selecting the initial probability (prior distribution) for the tool life. In general, this initial probability: 1) can be constructed from any combination of theoretical considerations, previous experimental results, and expert opinions; and 2) should be chosen to be as informative as possible using the experimenter's belief. In this study, the prior distribution was based on training tool life experiments.

\subsection{Training experiments}

The experimental steps followed to collect the tool wear data for selected turning operations are described in this section. The tool was a carbide insert and the workpiece material was MS309 steel. The initial outer diameter of the steel workpiece was $174.6 \mathrm{~mm}$. The depth of cut was 4.1 $\mathrm{mm}$. A single pass was defined as a single cut of length $139.7 \mathrm{~mm}$ with a 63.4 degree chamfer at the end of each cut. The spindle speed was varied to maintain constant cutting speed with reducing workpiece diameter as additional cuts were completed. A digital microscope (60× magnification) was used to image the flank and rake surfaces within the lathe enclosure to avoid removing the insert from the tool holder during the wear testing. The wear status of the tool was recorded after each pass and the calibrated digital images were used to identify the flank wear width $(F W W)$. The spindle power was monitored using a load control PH3A Hall effect power meter installed in the electrical cabinet of the lathe. As noted, the power sensor is installed remotely from the machining location and is non-intrusive in nature. Three tests were completed at various cutting speed, $V$, and feed rate, $f_{r}$, values: $\left\{V=153.6 \mathrm{~m} / \mathrm{min}, f_{r}=0.51 \mathrm{~mm} / \mathrm{rev}\right\}$, $\left\{V=192.01 \mathrm{~m} / \mathrm{min}, f_{r}=0.61 \mathrm{~mm} / \mathrm{rev}\right\}$ and $\{V=230.42$ $\left.\mathrm{m} / \mathrm{min}, f_{r}=0.51 \mathrm{~mm} / \mathrm{rev}\right\}$. Tool life was defined as the time required for the flank wear width $(F W W)$ to reach $0.5 \mathrm{~mm}$. Figure 1 shows the variation of $F W W$ with the number of passes for each test conditions.

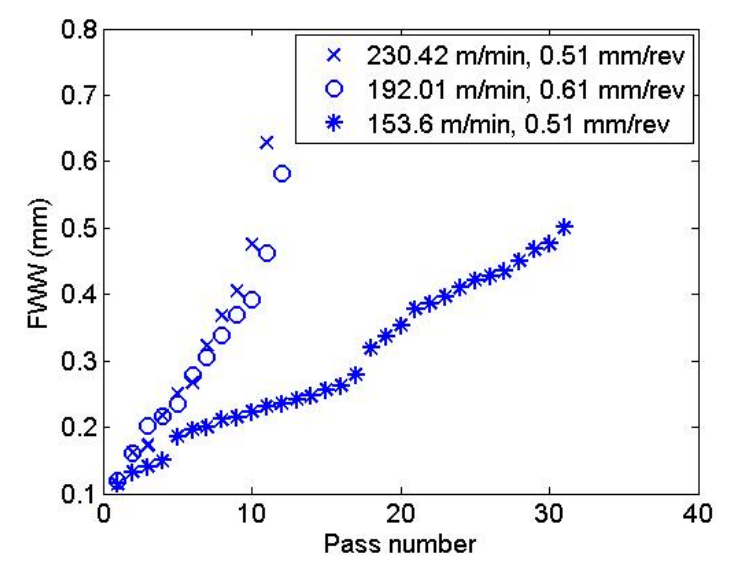

Figure 1. Variation of $F W W$ with the number of passes.

As expected, tool life reduces for higher speeds and feed rates. The spindle power was monitored over a 6 second cutting interval during a steady-state cutting period in each pass. The power features such as root mean square, $p_{r m s}$, average, $p_{a}$, standard deviation, $p_{\text {std }}$, and skewness, $p_{\text {skew }}$, in the time domain were calculated to evaluate their variation with tool life. The variation in power root mean square, average, standard deviation, and skewness as a function of 
$F W W$, is shown in Figures 2, 3, 4 and 5, respectively. The root means square $(R M S)$ was found to be sensitive tool wear. Figure 6 show the variation of RMS power with pass number.

As shown in Figures 2 and 6, the RMS power also depends on the speed and feed values. This implies that a large number of training experiments would be required to use the $R M S$ power values for tool life predictions at different speed and feed conditions. To eliminate the dependence of speed and feed on the RMS power values, the increment from the nominal was considered. The nominal value was determined from a measurement during the first pass. Figure 7 shows the variation of the increment of RMS power, denoted by $p_{r m s_{i}}$, as a function of $F W W$ (top) and number of passes (bottom) for all cutting conditions. [The additional subscript $i$ indicates that the values are calculated as increments from the nominal value.] The nominal value is defined as the value after the first pass. The purpose for this method is to enable the same scale to be used across all feed and speed conditions. The method is also employed in commercial tool condition monitoring systems.

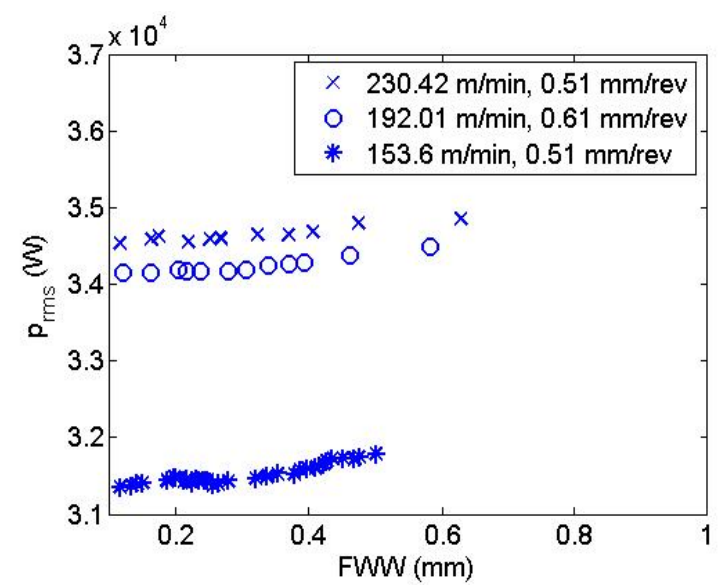

Figure 2. Variation of RMS power with FWW.

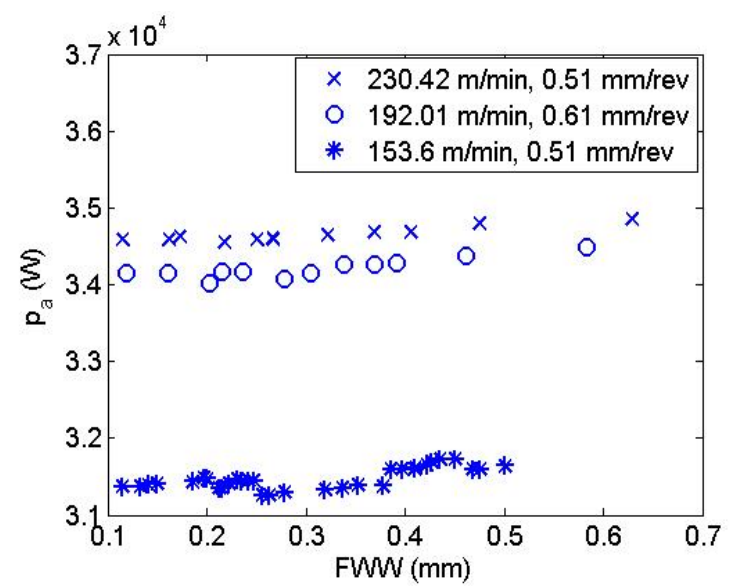

Figure 3. Variation of average power with $F W W$.

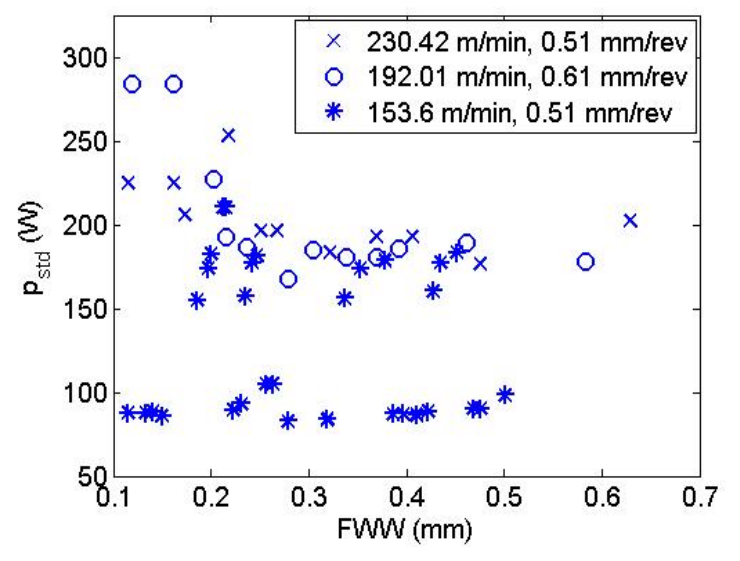

Figure 4. Variation of power standard deviation with $F W W$.

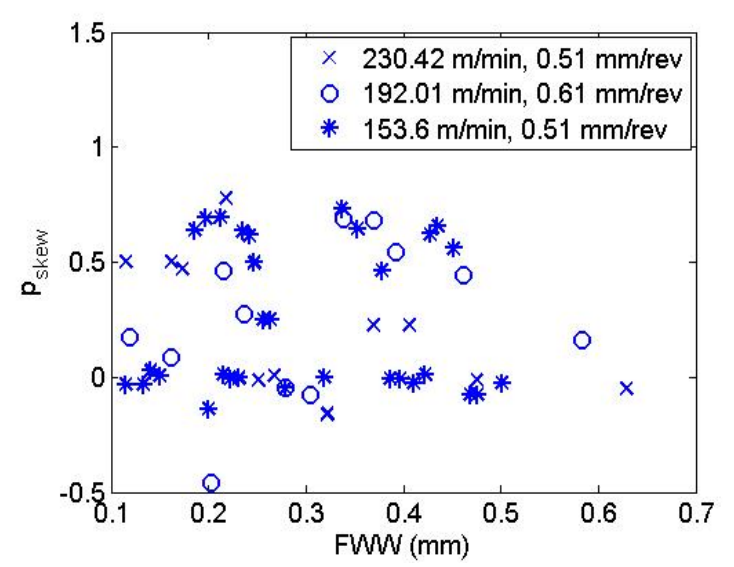

Figure 5. Variation of power skewness with $F W W$.

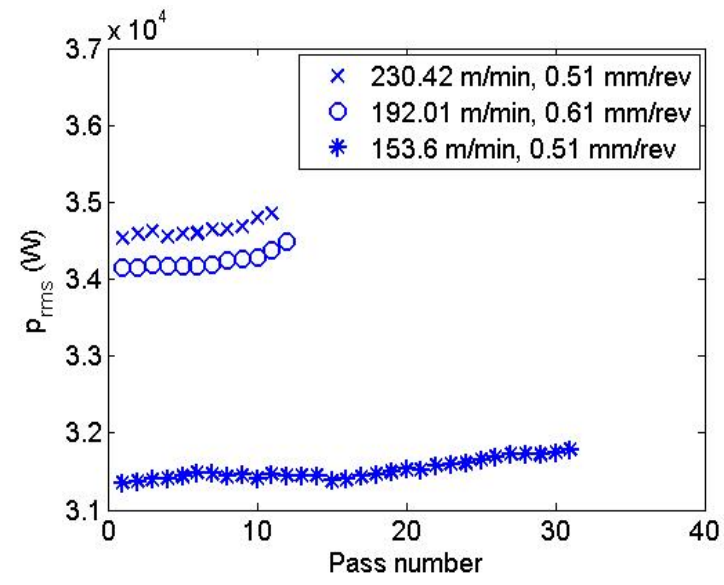

Figure 6. Variation of RMS power with the number of passes.

The following observations were made from Figure 7. First, the increase in RMS power over the nominal value was influenced by $F W W$. Second, the value of $p_{r m s_{i}}$ at the end of tool life $(F W W=0.5)$ was between $300 \mathrm{~W}$ and $400 \mathrm{~W}$. Finally, $p_{r m s}$ shows a non-linear increase with the number 
of passes which was best approximated by a second-order fit $\left(\mathrm{R}^{2}=0.75\right)$.
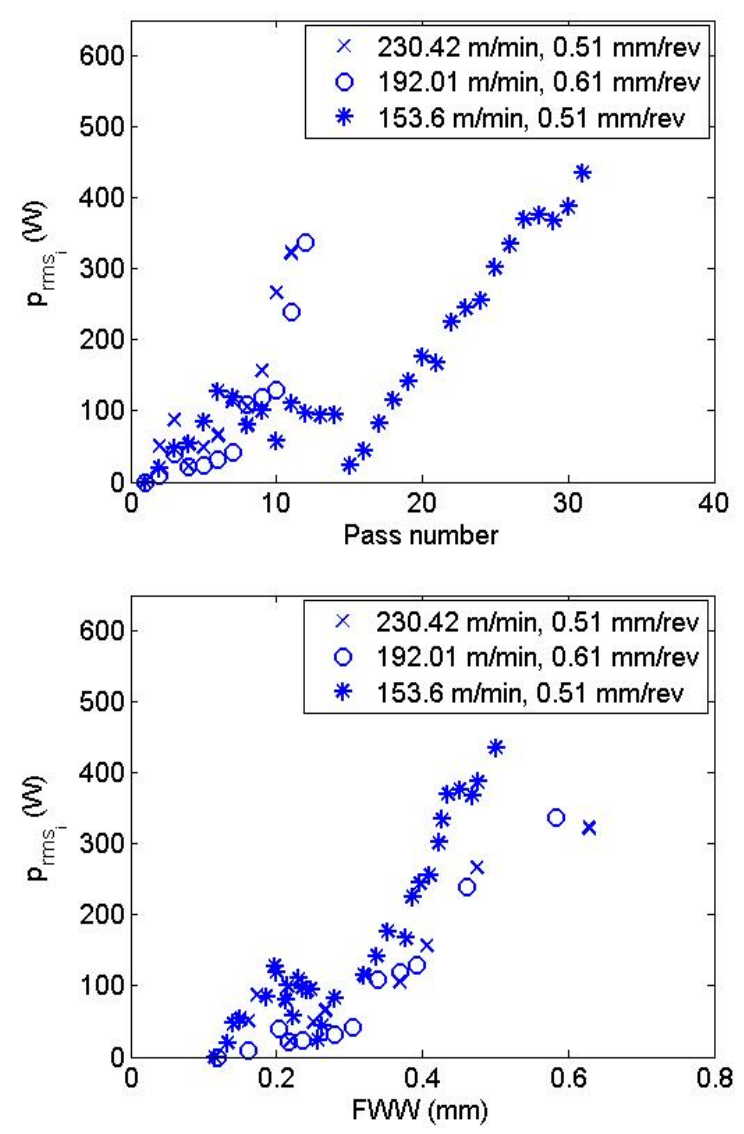

Figure 7. Increase in RMS power from the nominal value as a function of number of passes (top) and $F W W$ (bottom).

\section{BAYESIAN INFERENCE USING THE RANDOM SAMPLE PATH METHOD}

Bayesian inference provides a rigorous mathematical framework for belief updating about an unknown variable when new information becomes available. In this study, the tool remaining useful life was estimated using a random sample path method for Bayesian updating. As shown in Figure 7, $p_{r m s_{i}}$ is sensitive to tool wear and was therefore used to perform the Bayesian updating. Although a single sensor metric was used in this study, the method can be extended to include data from multiple sensors. The $p_{r m s_{i}}$ values increase with $F W W$ and, therefore, the number of passes. As noted, the growth in $p_{r m s_{i}}$ with the number of passes is non-linear and was approximated using a secondorder least squares fit. The random sample path method for Bayesian updating proceeds by generating sample $p_{r m s_{i}}$ growth curves, each of which represents the true $p_{r m s_{i}}$ growth curve with some probability. These sample paths were used as the prior for Bayesian inference. In this work, it was (initially) assumed that each sample curve was equally likely to be the true curve. This implies that if $N$ sample $p_{r m s_{i}}$ growth curves, or sample paths, are generated, each represents the true $p_{r m s_{i}}$ growth curve with an equal prior probability of $1 / N$. The prior probability of the sample paths was updated by applying Bayes' rule to $p_{r m s_{i}}$ measurements. Uncertain event $A$ is defined as the event when path is equal to the true $p_{r m s_{i}}$ growth and the $p_{r m s_{i}}$ measurement is the experimental result $B$. As noted, the prior probability that a given path is the true $p_{r m s_{i}}$ growth curve was assumed to be $1 / N$ before any $p_{r m s_{i}}$ measurement was completed since each path was considered equally likely to be the true $p_{r m s_{i}}$ growth curve. The product is normalized so that the sum of the probabilities of all sample paths is equal to one.

\subsection{Establishing the prior}

There is uncertainty in the growth of $p_{r m s_{i}}$ as a function of the number of passes and the tool life. The growth was approximated using a second-order polynomial shown in Eq. 2.

$$
p_{r m s_{i}}=a \times t^{2}+b \times t+c
$$

where $a, b$, and $c$ are the second order polynomial coefficients and $t$ is time in number of passes. This prior incorporated uncertainty (represented mathematically using the standard deviation) in the measurement due to both noise and the uncertainty in the second-order assumption. The combined measurement uncertainty level was set at 10 W. The prior random $p_{r m s_{i}}$ growth curves were generated using three points (for the second-order polynomial): one point at the end of the first pass, a second at the end of life, and a third intermediate point after the initial wear-in portion of the overall wear behavior. Note that the prior sample paths generation is based on the training experiments alone; therefore, a second order polynomial was found to fit the training data. In cases where additional data or expert information is available, empirical models or alternative statistical methods can be used to generate prior sample paths.

For the first point, the increment over the nominal value at the end of the first pass was assumed to follow a normal distribution with a mean of $0 \mathrm{~W}$ and a standard deviation of $10 \mathrm{~W}, N(0,10)$, where $N$ denotes a normal distribution and the numbers in the parenthesis identify the mean and standard deviation, respectively. For the second point, the $p_{r m s_{i}}$ value at the end of tool life, $p_{t}$, was assumed to be between $300 \mathrm{~W}$ and $400 \mathrm{~W}$ with equal probability, $U(300$, 400), where $U$ denotes a uniform distribution and the numbers in the parenthesis give the minimum and maximum values, respectively. Additionally, the uncertainty in tool life, denoted by $t_{\text {life }}$, identified by the limiting $F W W$ at some number of passes was incorporated by assuming a normal distribution. Note that the tool life distribution is a function 
of the speed and feed rate values. To illustrate, the tool life at $\{153.6 \mathrm{~m} / \mathrm{min}, 0.51 \mathrm{~mm} / \mathrm{rev}\}$ was assumed to be normally distributed with a mean of 31 passes (which was the tool life observed in the training experiment) with a standard deviation of 2 passes, $N(31,2)$. For the third point, the $p_{r m s_{i}}$ value at the end of the initial wear-in stage, defined by a $F W W$ of $0.25 \mathrm{~mm}$, was assumed to be equally likely between $20 \mathrm{~W}$ and $100 \mathrm{~W}, U(20,100)$. The time at the end of the initial wear stage was assumed to be uniformly distributed between 0.2 and 0.6 times the tool life value, denoted by $t_{\text {life }} \times U(0.2,0.6)$. The initial wear time and the $p_{r m s_{i}}$ values corresponding to the initial wear can be determined by the user from the data provided in Figure 7. Table 1 summarizes the distributions for the points used to fit the second order polynomial.

Table 1. Distributions for the points used to fit the second order polynomial.

\begin{tabular}{|l|l|l|}
\hline Point \# & $t$ & $p_{r m s_{i}}$ \\
\hline 1 & 0 & $N(0,10)$ \\
\hline 2 & $N(31,2)$ & $U(300,400)$ \\
\hline 3 & $t_{\text {life }} \times U(0.2,0.6)$ & $U(20,100)$ \\
\hline
\end{tabular}

The sample paths were generated as follows. First, random samples were drawn for the initial $p_{r m s_{i}}$ value, the $p_{r m s_{i}}$ value at the end of initial wear value, and the $p_{r m s_{i}}$ value at the end of tool life from the prior distributions. Next, random samples were drawn for the number of passes at the end of initial wear stage and end of tool life from the prior distributions. The time for the initial $p_{r m s_{i}}$ was one pass. A second-order least squares fit was performed for the three values of $p_{r m s_{i}}$ and number of passes. The procedure was repeated for $1 \times 10^{4}$ sample curves. An alternate method for generating the sample paths is to specify distributions for the second-order fit coefficients, for example. Figure 8 shows 10 sample curves for $\{153.6 \mathrm{~m} / \mathrm{min}, 0.51 \mathrm{~mm} / \mathrm{rev}\}$ with a distribution of $t_{\text {life }}$ taken as $N(31,2)$. Note that the values of $p_{r m s_{i}}$ can be negative as the values are expressed as increment over the nominal value. The negative $p_{r m s_{i}}$ values imply that the $p_{r m s}$ curves shown in Figure 6 need not be monotonically increasing due to random noise and uncertainty in the measurements. Figure 9 shows the prior cumulative distribution function (cdf) for $p_{r m s_{i}}$ as a function of the number of passes using the second-order model. The prior cdf gives the probability, $\mathrm{P}\left(p_{r m s_{i}}\right)$, that $p_{r m s_{i}}$ will be less than the selected value as a function of the number of passes. This is the gray scale value in Figure 9. Note that since $t_{\text {life }}$ is dependent on the speed and feed values, the prior generated is specific to the $\{153.6 \mathrm{~m} / \mathrm{min}$, $0.51 \mathrm{~mm} / \mathrm{rev}\}$ test condition. For different cutting conditions, the $t_{\text {life }}$ distribution will change, which, in turn, will change the prior cdf. The remaining distributions for the initial $p_{r m s_{i}}$ value, the $p_{r m s_{i}}$ value at the end of initial wear value, and the $p_{r m s_{i}}$ value at the end of tool life and the time at the end of the initial wear stage are independent of the speed and feed values. To illustrate, Figure 10 shows the cdf of $p_{r m s_{i}}$ at 20 passes. From Figure 10, the probability that $p_{r m s_{i}}$ is less than 220 after 20 passes is 1 , whereas the probability that $p_{r m s_{i}}$ is less than 110 after 20 passes is 0.53 .

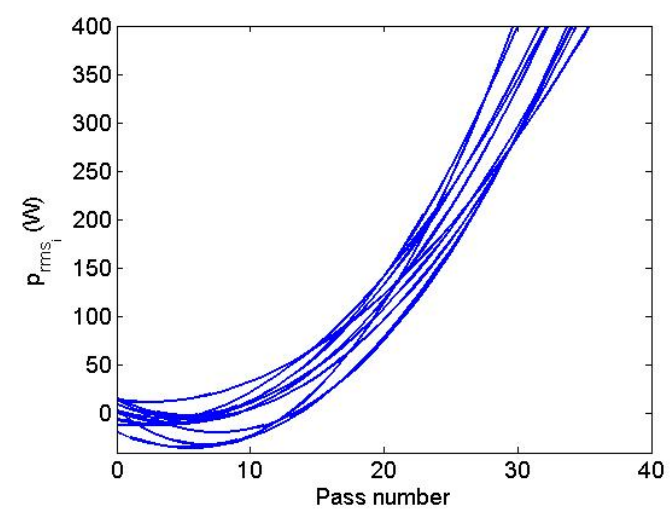

Figure 8. 10 sample $p_{r m s_{i}}$ growth curves.

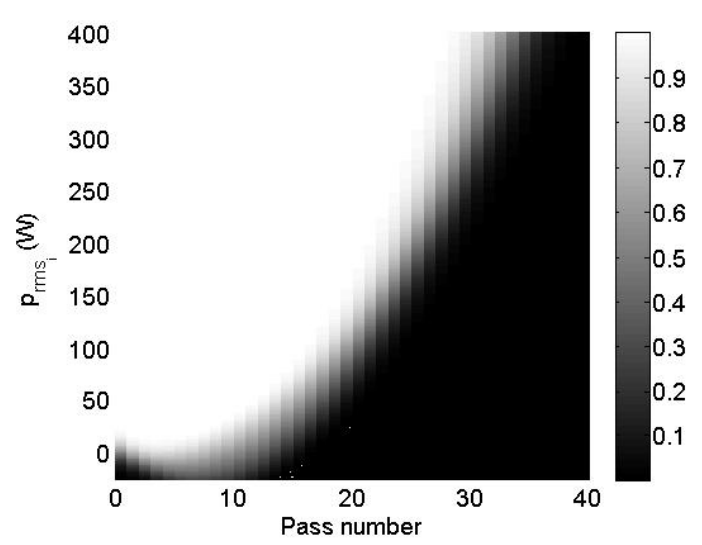

Figure 9. Prior cdf of $p_{r m s_{i}}$ using the second-order model. The gray scale color bar denotes the probability that $p_{r m s_{i}}$ will be less than the selected value.

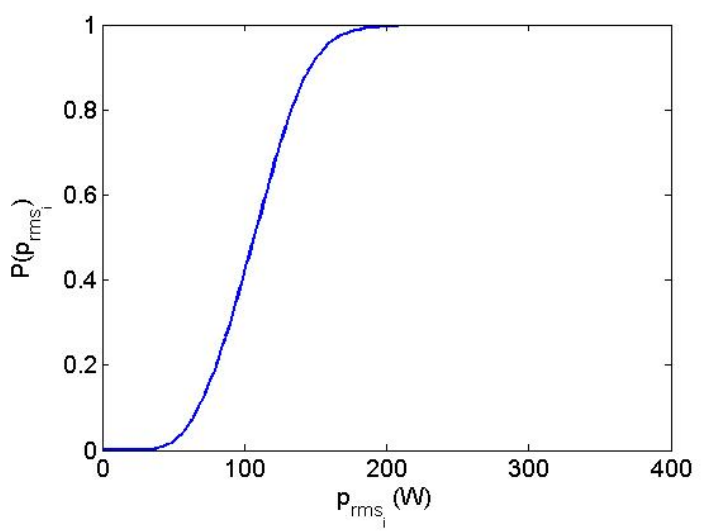

Figure 10. Prior cdf of $p_{r m s_{i}}$ at 20 passes. 


\subsection{Likelihood}

The probability of the sample $p_{r m s_{i}}$ growth curves was updated using $p_{r m s_{i}}$ measurements and Bayes' rule. The likelihood function incorporates the uncertainty in the $p_{r m s_{i}}$ measurement and the assumed second-order model. The likelihood is selected as Gaussian as a consequence of the central limit theorem. A non-normalized Gaussian distribution was used as the likelihood in this study. See Eq. 3:

$$
l=e^{\frac{-\left(p-p_{m}\right)^{2}}{k}},
$$

where $l$ is the likelihood value, $p_{m}$ is the measured $p_{r m s_{i}}$ value, $p$ is the $p_{r m s_{i}}$ value for a sample curve at the experimental spindle speed, and $k$ depends on the tool wear uncertainty. Because the likelihood function is expressed as a non-normalized normal distribution, $k=2 \sigma^{2}$, where $\sigma$ is the standard deviation of $p_{r m s_{i}}$; it represents the uncertainty in the $p_{r m s_{i}}$ measurement and the assumed second-order model. The likelihood function describes how likely it is that the $p_{r m s_{i}}$ measurement result would be obtained at a particular number of passes, given that the sample $p_{r m s_{i}}$ growth curve is the true curve. If the $p_{r m s_{i}}$ growth curve value is near the measurement result, then the likelihood value is high. Otherwise, it is low. The likelihood function can be interpreted as assigning weights from 0 to 1 to the sample curves; 0 implying not likely at all and 1 implying most likely. An increased uncertainty (higher $\sigma$ ) widens the likelihood function so that comparatively higher weights are assigned to sample curves further from the experimental result. Subsequently, larger uncertainty yields a more conservative estimate of tool life.

\subsection{Bayesian updating}

According to Bayes' rule, the posterior probability is the normalized product of the prior and likelihood. The prior probability for each path is $1 \times 10^{-4}$ and the likelihood value is determined using Eq. 3; the value of $\sigma$ was taken as $10 \mathrm{~W}$. The updated probabilities of the sample paths, calculated as the product of the prior and likelihood and normalized such that the sum of all probabilities of all sample paths is equal to unity, were used to determine the posterior $p_{r m s_{i}}$ cdf. To illustrate, consider an increment measurement of $p_{m}=70 \mathrm{~W}$ at 15 passes. Figure 11 shows the likelihood function for this result with $\sigma=10 \mathrm{~W}$. Figure 12 shows the updated cdf of the $p_{r m s_{i}}$ curves given $p_{m}=70 \mathrm{~W}$ at 15 passes. Note that the updated probabilities of the sample growth curves were used to calculate the posterior cdf. There is uncertainty in the value of $p_{r m s_{i}}$ at the end of tool life. Recall that it was assumed that the $p_{r m s_{i}}$ value at the end of tool life was equally likely to be between $300 \mathrm{~W}$ and $400 \mathrm{~W}$. The cdf value at a selected $p_{r m s}$ value gives the probability of $F W W$ being less than the critical $F W W(0.5 \mathrm{~mm})$, which is defined as the end of tool life. The probability that the tool $F W W$ is less than the critical $F W W$ is denoted by $p_{f}$. Each $p_{r m s_{i}}$ value has a probability distribution of $F W W$ being less than the critical $F W W$. To illustrate, Figure 13 shows the probability of $F W W$ being less that critical $F W W$ at $p_{t}=300$ $\mathrm{W}$ and $p_{t}=400 \mathrm{~W}$. Recall that $p_{t}$ denotes the $p_{r m s_{i}}$ value at the end of tool life. The 95\% RUL implies that there is a 0.05 probability of $F W W$ exceeding the critical $F W W$ value. Each $p_{t}$ value will have a different $95 \%$ value which will increase with the value selected.

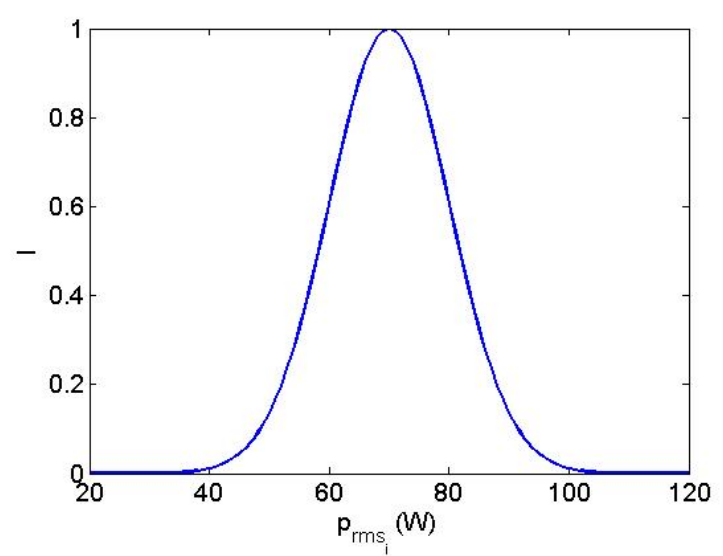

Figure 11. Likelihood function for $p_{m}=70 \mathrm{~W}$ and $\sigma=10 \mathrm{~W}$.

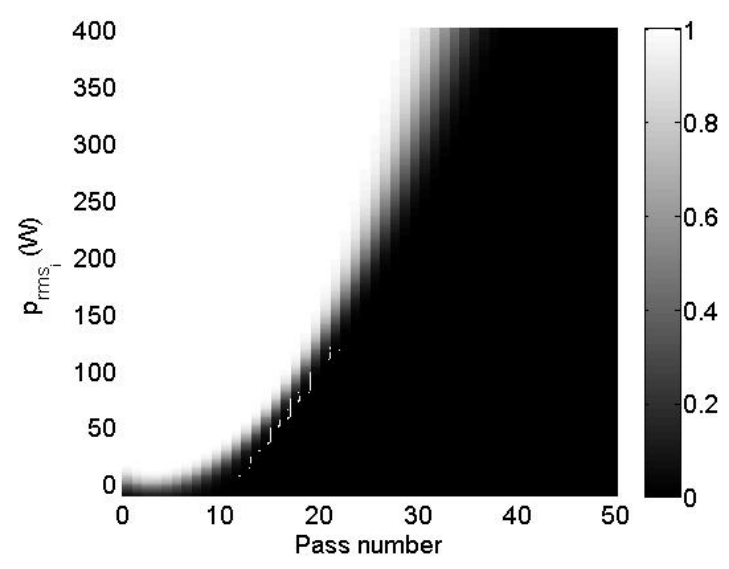

Figure 12. Posterior cdf of $p_{r m s_{i}}$ given $p_{m}=70 \mathrm{~W}$ at 15 passes.

From Figure 13, the $95 \%$ tool life value at $p_{t}=300 \mathrm{~W}$ is 25.5 passes; it is 28.9 passes at $p_{t}=400 \mathrm{~W}$. Note that the measurement is completed at 15 passes; therefore, the $95 \%$ $R U L$ values were 10.5 passes and 13.9 passes at $p_{t}=300 \mathrm{~W}$ and $p_{t}=400 \mathrm{~W}$, respectively. Since it was assumed that it is equally likely that the $p_{r m s}$ value is between $300 \mathrm{~W}$ and 400 W when the tool fails, the expected $R U L$ was calculated as the average value from all the threshold values. For example, if only $300 \mathrm{~W}$ and $400 \mathrm{~W}$ values were considered, the 95\% RUL was 12.2 passes. The Bayesian updating procedure for RUL predictions is summarized as follows. 
First, training experiments were performed to observe and identify trends in the power signals as a function of machining time and tool wear. Second, random sample power RMS growth curves were generated based on the training experiments. Although a second order polynomial model was used in this study, alternative methods such as using model parameter distributions can be used. Third, a prior probability was assigned for each sample growth curve to be the true curve. In this study, each sample path was assumed to be equally likely to be the true path a priori. Fourth, the prior remaining life was calculated based on the prior probabilities of sample curves. Fifth, power RMS was measured during machining and a likelihood value was assigned for all sample paths based on the measurement. Sixth, the posterior probabilities of the sample growth curves were calculated using Bayes' rule. Finally, the posterior remaining life was calculated using posterior probabilities of the sample paths. The posterior probabilities become prior for the next update and so on. The updating procedure is repeated for multiple measurements using the procedure described.

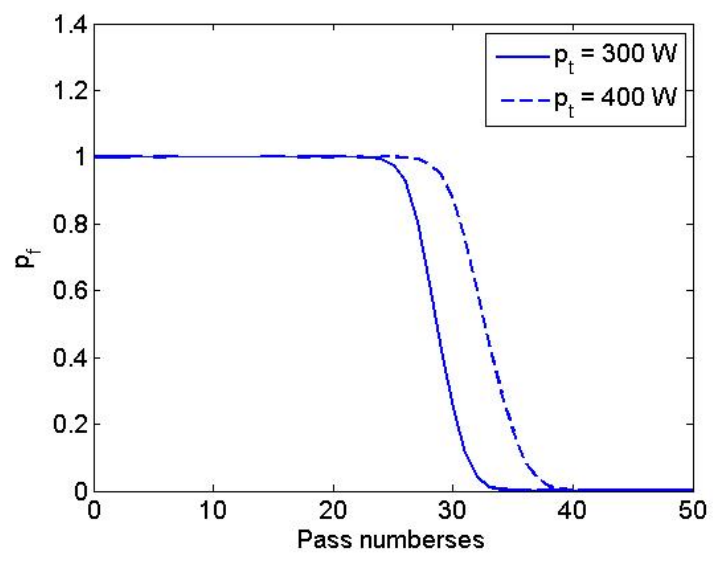

Figure 13. Probability of $F W W$ being less that critical $F W W$ at $p_{r m s_{i}}=300 \mathrm{~W}$ and $p_{r m s_{i}}=400 \mathrm{~W}$.

\section{RUL PREDICTIONS}

Bayesian inference using a random sample path approach was used to predict remaining useful tool life for new test conditions: $\left\{V=192.01 / \mathrm{min}, f_{r}=0.51 \mathrm{~mm} / \mathrm{rev}\right\}$. Two tool life tests were performed. Note that the speed, feed combination is different than the training experiments. The procedure for the tests was the same as described previously and the other operating parameters were the same as for the training experiment. The $F W W$ and $p_{r m s}$ were measured after every pass. For subsequent passes, $p_{r m s}$ was calculated as an increment over the nominal value measured at the first pass. The first step in applying Bayesian inference is to determine the prior. Recall that the distribution for the initial $p_{r m s_{i}}$ value was $N(0,10)$, the $p_{r m s_{i}}$ value at the end of initial wear value was $U(20,100)$, the $p_{r m s_{i}}$ value at the end of tool life was $U(300,400)$, and the distribution for the end of initial wear stage was $t_{\text {life }} \times U(0.2,0.6)$, as described in Section 3.1. As noted, these distributions are independent of the speed and feed conditions as seen from the training data. The distribution of tool life is dependent on the speed and feed values and must be modified. As shown in training experiments, the tool life for $\left\{V=153.6 / \mathrm{min}, f_{r}=0.51\right.$ $\mathrm{mm} / \mathrm{rev}\}$ was found to be 31 passes and $\{V=230.42 / \mathrm{min}$, $\left.f_{r}=0.51 \mathrm{~mm} / \mathrm{rev}\right\}$ was found to be 11 passes (see Figure 1). Although tool life reduces exponentially with cutting speed, the tool life at $\left\{V=192.01 / \mathrm{min}, f_{r}=0.51 \mathrm{~mm} / \mathrm{rev}\right\}$ was assumed to be normally distributed with a mean of 19 passes and a standard deviation of 2 passes, $N(21,2)$, using linear interpolation. The variance of the distribution takes into account the uncertainty in the linear approximation. Figure 14 shows the prior cdf of $p_{r m s_{i}}$ at $\{V=192.01 / \mathrm{min}$, $\left.f_{r}=0.51 \mathrm{~mm} / \mathrm{rev}\right\}$. Figures 15 and 16 display $F W W$ and $p_{r m s_{i}}$ as a function of the number of passes for the first test, respectively. The tool life was found to be 16 passes.

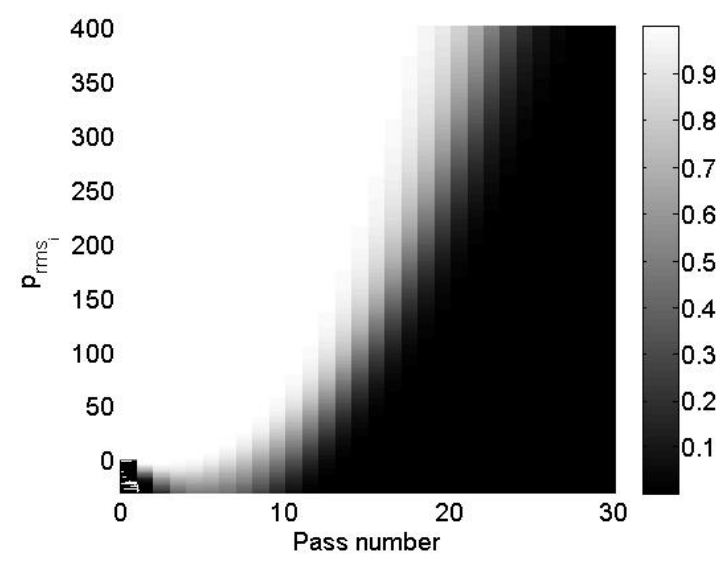

Figure 14. Prior cdf of $p_{r m s_{i}}$ at $\left\{V=192.01 / \mathrm{min}, f_{r}=0.51\right.$ $\mathrm{mm} / \mathrm{rev}\}$.

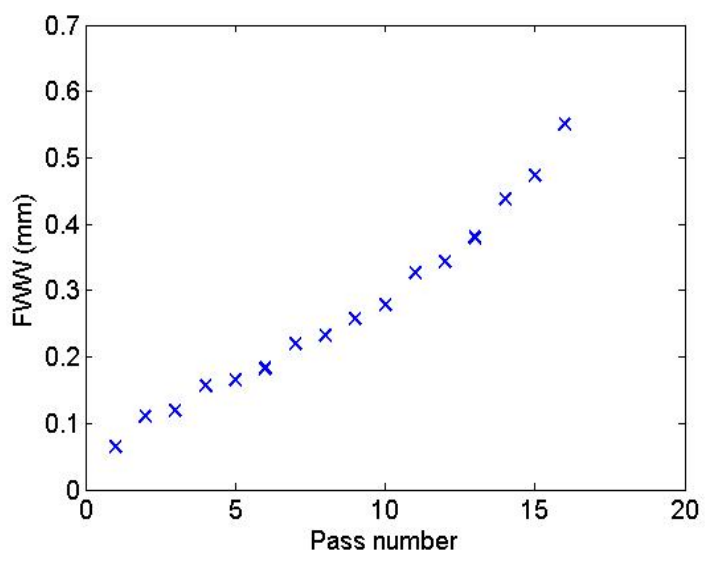

Figure 15. Variation in $F W W$ with the number of passes. 


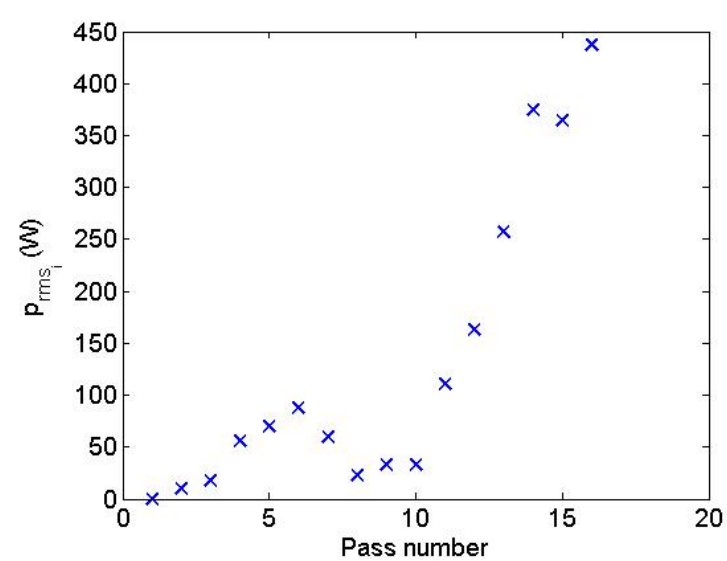

Figure 16. Variation in $p_{r m s}$ with the number of passes.

The values of $p_{r m s_{i}}$ shown in Figure 16 were used to update the prior probabilities of sample paths. The value of $\sigma$ was assumed to be $10 \mathrm{~W}$. The updated probabilities of sample paths were used to calculate the posterior cdf of $p_{r m s_{i}}$. From the updated probabilities of the sample $p_{r m s_{i}}$ growth curves, the $R U L$ of the tool before the FWW reaches the critical value was calculated. As noted, a 95\% RUL estimate implies that there is a 0.05 probability of the tool wear exceeding the critical $F W W$ limit of $0.5 \mathrm{~mm}$. Each $p_{r m s_{i}}$ measurement updates the RUL estimates. For the predictions, the $p_{t}$ values were discretized into 101 levels between $300 \mathrm{~W}$ and $400 \mathrm{~W}$ and the RUL estimate was taken as the average value.

Figure 17 shows the posterior cdf of $p_{r m s_{i}}$. Figure 18 shows the $95 \%$ RUL of the tool after each measurement and the true remaining life calculated from the observed tool life value (16 passes). The $95 \% R U L$ predicts a tool life of -1.6 passes at the end of tool life. A negative RUL estimate implies that the tool is past the end of tool life by 1.6 passes. The initial 95\% RUL prediction is not conservative due to the uncertainty in the $p_{r m s_{i}}$ second-order growth model in the prior distribution. The estimate approaches the true life and over predicts in the range of 7 to 10 passes. This is because $p_{r m s}$ values decrease from pass number 7 to pass number 10 . These assign a higher likelihood to sample paths predicting a tool life greater than 20 passes. However, subsequent measurements greater than $100 \mathrm{~W}$ reduce the $R U L$ predictions to a conservative value. The final estimate is conservative as the $p_{r m s_{i}}$ at the end of tool life is 437.2 $\mathrm{W}$. Recall that it was assumed that the $p_{r m s_{i}}$ value at the end of tool life was equally likely to be between $300 \mathrm{~W}$ to 400 W. Additional testing was completed using the same procedure as previously described. Figures 19 and 20 show $F W W$ and $p_{r m s_{i}}$ as a function of the number of passes for the second test, respectively. The tool life was found to be 17 passes. Again, the $p_{\text {rms }}$ value was measured after each pass and the increment over the nominal value was considered after the first pass. The measured $p_{r m s_{i}}$ values were used to update the prior probabilities of the sample $p_{r m s_{i}}$ growth curves and estimate the 95\% RUL. Figure 21 shows the posterior cdf $p_{r m s_{i}}$. Figure 22 shows $R U L$ predictions for the second test. The 95\% RUL predict a tool life of -3.7 passes at the end of tool life. The predictions are conservative due to $p_{r m s_{i}}$ values being greater than $300 \mathrm{~W}$ at the end of $13^{\text {th }}$ pass $(F W W=0.355 \mathrm{~mm})$.

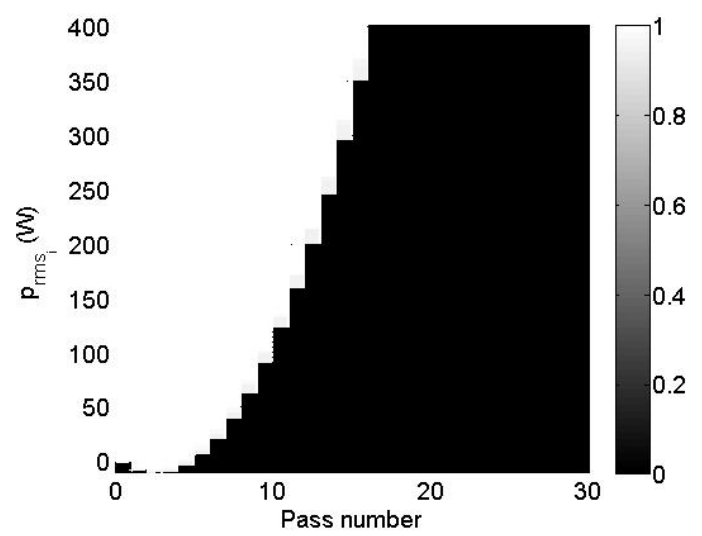

Figure 17. Posterior cdf of $p_{r m s_{i}}$.

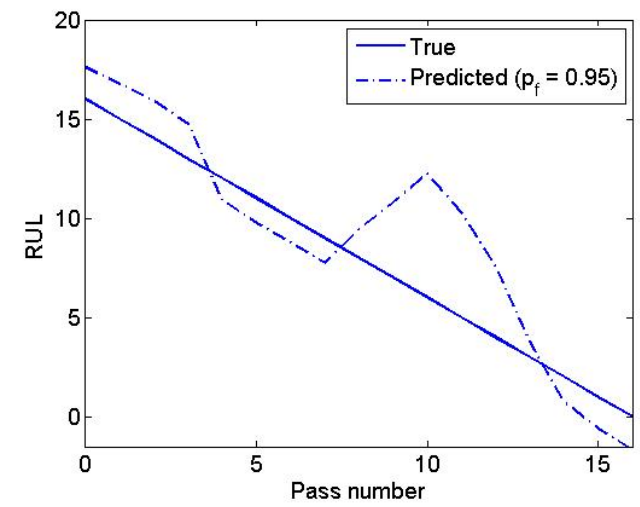

Figure 18. RUL predictions comparison with the true remaining life.

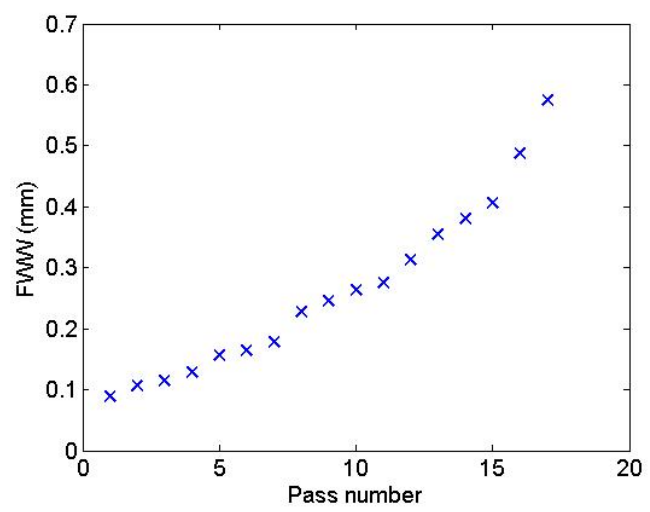

Figure 19. Variation in $F W W$ with the number of passes. 


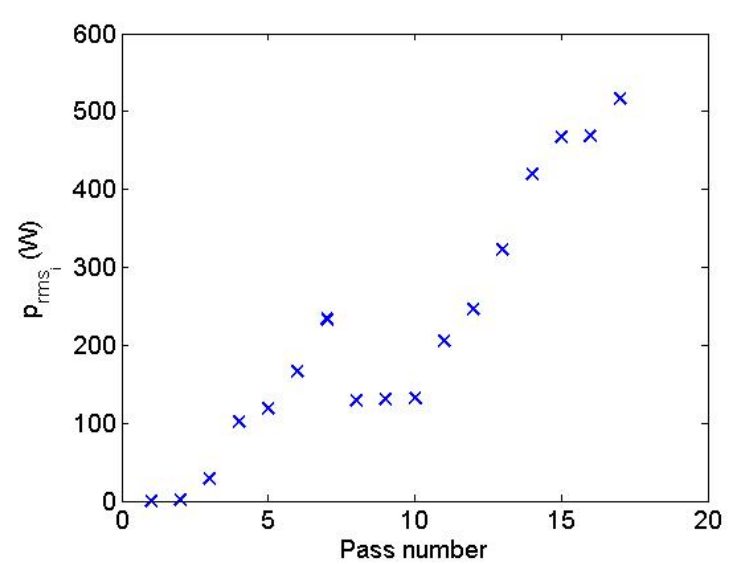

Figure 20. Variation in $p_{r m s_{i}}$ with the number of passes.

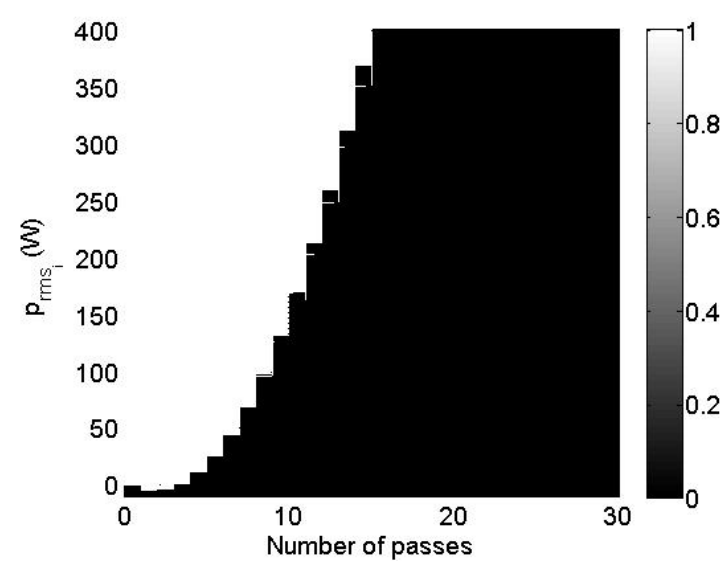

Figure 21. Posterior cdf of $p_{r m s_{i}}$.

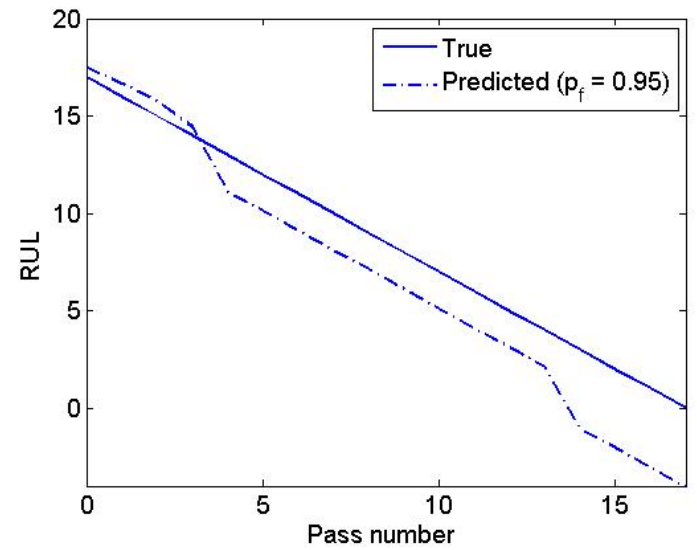

Figure 22. RUL predictions.

The advantage of the method is that the prior estimates on the threshold values and tool life can be continuously updated based on observed experimental evidence. For example, for the second test the $p_{r m s}$ value can be assumed to be equally likely between $400 \mathrm{~W}$ and $500 \mathrm{~W}$ at the end of tool life. To illustrate, the $R U L$ predictions were repeated assuming that $p_{r m s}$ value to be equally likely between 400 $\mathrm{W}$ and $500 \mathrm{~W}$ based on the results of the first test. The RUL predictions estimate a tool life of -2.2 passes at the end of tool life as compared to -3.7 passes (see Figure 22). Figure 23 compares the two results. In general, the predictions are better if the tool life occurs at the $p_{r m s_{i}}$ value within the interval considered, which can be updated for subsequent tests. The sample prior curves can be generated using the updated values for every test. This would improve the predictions for subsequent tests. Future work will focus on improving the prior by using experimental result data from tests. The proposed method takes into account the uncertainty in the threshold value and the tool life as opposed to a deterministic threshold value of $p_{r m s}$ which will result in an erroneous alarm for a worn tool obtained from a single outlier measurement (Karandikar, McLeay, Turner, \& Schmitz, 2013).

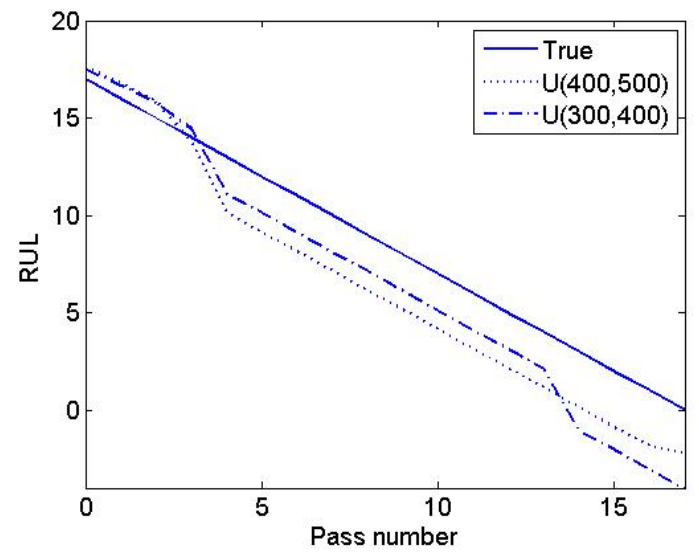

Figure 23. RUL predictions for different distributions of $p_{r m s_{i}}$ at the end of tool life.

\section{CONCLUSIONS}

A random sample path method of Bayesian updating for predicting remaining useful tool life was presented. The root mean square of the time domain spindle power was found to be sensitive to tool wear. Random sample growth curves for the root mean square power were generated and the probability of each representing the true curve was updated using measurements. The updated probabilities of the sample curves were used to predict the remaining useful tool life. The method offers many advantages. First, it incorporates uncertainties in tool life and the power sensor value at the end of tool life. Therefore, the method is robust to outlier points and more reliable than methods that rely on a deterministic threshold sensor value. The method incorporates prior information and does not require a large training data set. The prior information can also be improved after each test. Second, the method is computationally inexpensive and can be incorporated for real-time predictions. To illustrate, using $\mathrm{Matlab}^{\mathrm{TM}}$ on an 
Intel i5 processor, the computation described here takes 1 second. An alternative method for Bayesian inference is to update the distribution of the model coefficients (in this case, coefficients of the second order polynomial used to generate the sample paths). However, the method requires sampling from posterior joint distributions in case of nonconjugate distributions, which may be computationally expensive. Without the loss of generality, the random sample path method described is applicable for any nonconjugate likelihood such as triangular distribution. Future work will try to compare and quantify various Bayesian updating methods for remaining useful life predictions. Third, the remaining useful life percentile (e.g., 95\%) can consider user preferences and applications. For example, in an application using expensive parts/tooling, the user can select a conservative RUL percentile (say 99\%). On the other hand, for low cost applications, such as roughing, the user can select a low percentile (say 90\%). The optimum percentile can be calculated using an expected cost formulation. The risk preferences of the user (risk neutral or risk averse) can also be incorporated. Future work will explore the optimum percentile calculations using decision theory principles.

\section{ACKNOWLEDGEMENT}

The authors gratefully acknowledge financial support from the National Science Foundation (CMMI-0927051 and CMMI-0926667) and General Dynamics-OTS. They would also like to thank E. Deane, M. Hernandez, and D. Giesecke, University of Central Florida, and H.S. Kim, University of Florida, for their help with the tool wear experiments.

\section{REFERENCES}

Constantinides, N., and Bennett, S. (1987), An investigation of methods for on-line estimation of tool wear, International Journal of Machine Tools and Manufacture, 27 (2), pp. 225237.

Dey, S., and Stori, J. A. (2005), A Bayesian network approach to root cause diagnosis of process variations, International Journal of Machine Tools and Manufacture, 45(1), pp. 75-91.

Dimla E. Snr. (2000), Sensor signals for tool-wear monitoring in metal cutting operations - a review of methods, International Journal of Machine Tools and Manufacture, 40, pp. 10735-1098.

Elangoavn, M., Ramachandran, K. I., and Sugmaran, V. (2010), Studies on Bayes classifier for condition monitoring of single point carbide tipped tool based on statistical and histogram features, Expert Systems with Applications, 37, pp. 2059:2065.

Jawahir, I., Li, P., Gosh, R., and Exner, E. (1995), A new parametric approach for the assessment of comprehensive tool wear in coated grooved tools, Annals of the CIRP, 44, pp. 49-54.

Karandikar, J., McLeay, T., Turner, S., and Schmitz, T. (2013), Remaining useful tool life predictions using Bayesian inference, Manufacturing Science and Engineering Conference, MSEC2013-1152, 2013, June 10-14, Madison, WI, USA.

Karandikar, J., Schmitz, T., and Abbas, A. (2010), Tool life prediction using Bayesian updating, Transactions of the NAMRI/SME, 39.

Li, P., Stein, D., Gosh, R., and Jawahir, I. (1997), Engaged cutting edge effects on tool-wear and tool-life in turning operations using grooved cutting tools, Manufacturing Science and Technology, 2, pp. 277-284.

Prickett P. W., and Johns, C. (1999), An overview of approaches to end milling tool monitoring, International Journal of Machine Tools and Manufacture, 39, pp. 105122.

Quinto, D. T. (1988), Mechanical property and structure relationships in hard coated carbide tools, Metals Technology, 9, pp. 60-75.

Rangwala, S., and Dornfeld, D. (1990), Sensor integration using neural networks for intelligent tool condition monitoring, ASME Trans. Journal of Engineering for Industry, 112 (3), pp. 219-228.

Ravindra,H. V., Srinivasa, Y.G. and Krishnamurthy, R. (1993), Modelling of tool wear based on cutting forces in turning, Wear, 169, pp. 25-32.

Taylor, F.W. (1906), On the Art of Cutting Metals, Transactions of the ASME, Vol. 28, pp. 31-248.

Tlusty, J. (2000), Manufacturing Process and Equipment, Prentice Hall, Upper saddle River, NJ, pp. 463.

\section{BIOGRAPHIES}

Jaydeep M. Karandikar is a $\mathrm{PhD}$ student under the guidance of Dr. Tony Schmitz in the Mechanical Engineering and Engineering Science Department at the University of North Carolina at Charlotte, Charlotte, NC, USA. He received his MS from the Mechanical and Aerospace Engineering Department at the University of Florida, Gainesville, FL, USA. At the University of Florida, he was the recipient of Outstanding International Student award and was inducted into Tau Beta Pi. He is the also the recipient of the 2013 Outstanding Graduate Research assistant award at the University of North Carolina at Charlotte. His research interests are manufacturing process modeling, tool wear, structural health and process monitoring, and application of decision analysis and Bayesian inference to manufacturing. His paper co-authored 
with Dr. Schmitz received the outstanding paper award at the North American Manufacturing Research Conference in 2010.

Ali E. Abbas received an M.S. in electrical engineering (1998); M.S. in engineering economic systems \& operations research (2001); $\mathrm{PhD}$ in management science and engineering (2004), and $\mathrm{PhD}$ minor in electrical engineering all from the school of engineering at Stanford University. His research interests include decision making under uncertainty, decision making with incomplete information and preferences, dynamic programming, information theory and applications of operations research tools in bioinformatics. He is the Art Davis Faculty Scholar in the Department of Industrial and Enterprise Systems Engineering at the University of Illinois at UrbanaChampaign. He joined UIUC in 2004, and previously worked as a Lecturer in the Department of Management Science and Engineering at Stanford University and in Schlumberger Oilfield Services, where he held several positions in Wireline logging, operations management, and international training. Dr. Abbas received the National Science Foundation Career Award in 2008 and the Decision Analysis Society Best Publication Award in 2011. He is associate editor for both the Operations Research and Decision Analysis journals of INFORMS and decision analysis area editor for the Institute of Industrial Engineers' IIE Transactions.

Tony L. Schmitz received his BS in Mechanical Engineering from Temple University in 1993, his MS in Mechanical Engineering from the University of Florida in 1996, and his $\mathrm{PhD}$ in Mechanical Engineering from the
University of Florida in 1999. He completed a post-doctoral appointment at the National Institute of Standards and Technology (NIST) and was then employed as a Mechanical Engineer from 1999-2002. During this time, he was also a lecturer at Johns Hopkins University. Schmitz accepted an appointment in the University of Florida's Department of Mechanical and Aerospace Engineering (UF MAE) in 2002 and joined the Department of Mechanical Engineering and Engineering Science at the University of North Carolina at Charlotte in 2011. His professional recognitions include: 2013 UNC Charlotte Lee College of Engineering Undergraduate Award in Teaching Excellence, 2012 Temple University Alumni Fellow, 2011 Sports Emmy Award (NBC Learn) for the Science of NFL Football video series, 2010 North American Manufacturing Research Institute/Society of Manufacturing Engineers (SME) Outstanding Paper, 2009 UF MAE Teacher of the Year, 2005 SME Outstanding Young Manufacturing Engineer award, 2004 Journal of Tribology Best Paper Award, 2003 Office of Naval Research Young Investigator Award, 2003 National Science Foundation CAREER Award, 1999 Measurement Science and Technology Highly Commended Article, 1999 National Research Council Postdoctoral Research Associateship (NIST), 1999 Temple University Gallery of Success Inductee, 1998 Department of Energy/National Academy of Engineering Integrated Manufacturing Predoctoral Fellowship, and 1994 National Science Foundation Graduate Traineeship. He also serves as an associate editor for the ASME Journal of Manufacturing Science and Engineering. 